\title{
Estudio de la competencia para el autocuidado de las personas en tratamiento renal sustitutivo con diálisis
}

\author{
Claudia Patricia Cantillo-Medina, Brigitte Vanessa Castro-Morea, Yury Hasbleydi Mosquera \\ Universidad Surcolombiana. Neiva. Huila. Colombia
}

Como citar este artículo:

Cantillo-Medina CP, Castro-Morea BV, Mosquera YH. Estudio de la competencia para el autocuidado de las personas en tratamiento renal sustitutivo con diálisis.

Enferm Nefrol. 2021 0ct-Dic;24(4):398-407

\section{Resumen}

Introducción: Las personas con enfermedad renal crónica dializadas demandan mayor cuidado por el manejo complejo de su condición, las múltiples complicaciones asociadas al tratamiento y la necesidad de asumir estilos de vida saludables, para garantizar la efectividad y seguridad de las terapias.

Objetivos: Determinar la competencia para el autocuidado de las personas en diálisis, así como su relación con las características sociodemográficas y clínicas.

Metodología: Se realizó un estudio observacional descriptivo de corte transversal, en una unidad renal del sur de Colombia. Instrumentos utilizados: Encuesta de caracterización para el cuidado de la persona con enfermedad crónica, Formulario de características clínicas y Cuestionario de Competencia para el cuidado versión-paciente.

Resultados: Se incluyeron un total de 200 personas dializadas, con una edad media de 59 $\pm 13,89$ años, el $50,5 \%$ mujeres, con pareja estable, apoyo para su cuidado, grado educativo primario, dedicados a labores del hogar, y nivel socioeconómico bajo. El 47,5\% evidenció alta competencia para su autocuidado, el $42 \%$ una competencia media y el $10,5 \%$ baja. Se identificaron asociaciones estadísticamente significativas de la com-

\section{Correspondencia:}

Claudia Patricia Cantillo-Medina

E-mail: claudiacantillol@hotmail.com petencia para el autocuidado con las variables asistencia puntual a terapia $(p=0,024)$ y adherencia farmacológica $(p=0,001)$.

Conclusiones: La población abordada se caracterizó por vivir en condiciones socioeconómicas que aumentan la vulnerabilidad de la persona dializada, en su mayoría contó con soporte para el cuidado, presentó nivel de competencia para el autocuidado entre alto y medio, relacionado con asistencia puntual a terapia y cumplimiento en adherencia farmacológica.

PALABRAS CLAVE: competencia clínica; insuficiencia renal crónica; dialisis; cuidado de enfermería; seguridad del paciente.

\section{Study of the self-care competence of people on renal replacement therapy with dialysis}

\section{Abstract}

Introduction: People with chronic kidney disease on dialysis require greater care due to the complex management of their condition, the multiple complications associated with treatment and the need to adopt healthy lifestyles in order to guarantee the effectiveness and safety of the therapies.

Objective: To determine the self-care competence of people on dialysis, as well as its relationship with sociodemographic and clinical characteristics. 
Methodology: A descriptive cross-sectional observational study was carried out in a renal unit in southern Colombia. Instruments used: Characterisation survey for the care of the person with chronic disease, clinical characteristics form and the patient-version of the Competence for Care Questionnaire.

Results: 200 dialysis patients were included, with a mean age of $59 \pm 13.89$ years, $50.5 \%$ women, with a stable partner, support for their care, primary education, dedicated to household chores, and low socioeconomic level. $47.5 \%$ showed high self-care competence, $42 \%$ medium competence and $10.5 \%$ low competence. Statistically significant associations of self-care competence with the variables punctual attendance to therapy $(p=0.024)$ and pharmacological adherence $(p=0.001)$ were identified.

Conclusions: The population studied was characterised by living in socioeconomic conditions that increase the vulnerability of the dialysed person, most of them had support for care, presented a high to medium level of competence for self-care, related to punctual attendance at therapy and compliance with pharmacological adherence.

KEYWORDS: clinical competence; chronic kidney disease; dialysis; nursing care; patient safety.

\section{Introducción}

El aumento de las enfermedades cardiovasculares, la diabetes mellitus tipo II, el envejecimiento y los estilos de vida de las personas, han influido de manera importante en la actual epidemiología de la enfermedad renal crónica $(E R C)^{1,2}$. La ERC está considerada como problema de salud pública a nivel mundial por su impacto en muchas poblaciones y sobrecarga de los servicios de salud $^{3}$, se estima que 850 millones de personas padecen de esta enfermedad, con aproximadamente 2,4 millones de muertes al año4.

Es una condición debilitante para la persona en la medida en que pierde de manera gradual y progresiva su función renal, y no cuenta con pronóstico de mejoría rápida, ${ }^{5,6}$; es un predictor de la disminución de la calidad de vida que afecta a la persona y a su familia de diferentes maneras ${ }^{7}$.

Esta enfermedad es compleja, costosa, se prolonga en el tiempo y requiere un gran apoyo en la atención, no solo de las entidades sanitarias sino también en la familia, ya que tiene que asumir una gran cantidad de tareas que lo ubica en una situación de gran vulnerabilidad, estrés y carga, y que incrementa, a su vez, el riesgo de padecer problemas físicos, emocionales, sociales, familiares y otros ${ }^{8}$. Las terapias de reemplazo renal (TRR) generan en la persona con diálisis múltiples demandas y problemas que la mayoría de las veces no pueden satisfacer ni solucionar por sí mismos, además no es posible resolverlas durante su estancia en la unidad renal, por lo cual requieren de una asistencia continua en el hogar?.

En este contexto la población vulnerable requiere mayor cuidado, por el manejo complejo de la condición de salud y las múltiples complicaciones asociadas al tratamiento ${ }^{10}$, es importante destacar que las terapias son efectivas y seguras cuando las personas realizan cambios en su estilo de vida como: adherencia al régimen terapéutico alimentación y medicación, cuidado con el acceso para diálisis, actividad física, y manejo del estrés ${ }^{7}$; deben asumir una serie de comportamientos que enmarcan y proyectan su adecuado autocuidado en todos los ambientes en los cuales el individuo se desarroIla.

Por lo expuesto anteriormente, el objetivo de este estudio fue determinar la competencia para el autocuidado de las personas en diálisis, así como su relación con las características sociodemográficas y clínicas.

\section{Material y Método}

Se llevó a cabo un estudio observacional descriptivo de corte transversal. El muestreo utilizado fue no probabilístico intencional. La información fue recolectada durante 6 meses en una Unidad Renal Fundación Nefrouros Neiva (Colombia). Los participantes cumplieron con los criterios de inclusión propuestos: mayor de 18 años, en tratamiento con diálisis crónica (hemodiálisis o diálisis peritoneal) por un período mayor a tres meses y aceptación voluntaria a participar en el estudio. Se excluyeron las personas en tránsito de otra unidad renal, fase aguda de fallo de la función renal y personas con demencia o alteraciones cognitivas registradas en la historia clínica.

Las variables de resultado del estudio fueron las características sociodemográficas: edad, género, estado civil, escolaridad, nivel socioeconómico, ocupación y contar con apoyo de un cuidador; variables clínicas: tipo 
de TRR, índice de masa corporal (IMC), presencia de edemas, objetivos de tensión arterial, asistencia puntual a terapia, mantenimiento de peso seco, adherencia a la toma de medicamentos, y la Competencia para el autocuidado de la persona en tratamiento con diálisis.

Para su valoración se utilizaron los siguientes instrumentos:

- Encuesta de caracterización para el cuidado de una persona con enfermedad crónica (GCPC-UN-P), compuesta de 22 ítems y tres dimensiones: 1) Las condiciones y perfil socio- demográfico de la persona con ERC. 2) La percepción de carga y apoyo. 3) Los medios de información y comunicación. Esta encuesta está desarrollada y validada para la caracterización de una persona con enfermedad crónica en el contexto de América Latina ${ }^{11}$. Incluye la escala de valoración funcional creada y empleada por Moskowitz en $1957^{12}$ (PULSES), se refiere

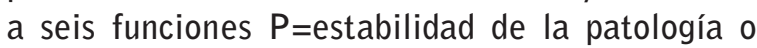
condición física, $\mathrm{U}=$ utilización de miembros superiores, $\mathrm{L}=$ locomoción o función de los miembros inferiores, $\mathrm{S}=$ función sensorial, $\mathrm{E}=$ =eliminación 0 control de esfínteres, $\mathrm{S}=$ capacidad de socializar; evalúa no solo la capacidad funcional aislada sino la capacidad para realizar actividades de la vida diaria. Además, contiene el Cuestionario corto de valoración del estado mental (SPMSQ-PFEIFFER), para la detección de deterioro cognitivo, que valora 4 parámetros: memoria a corto y largo plazo, orientación, información sobre hechos cotidianos y capacidad de cálculo ${ }^{13}$.

- Cuestionario de características clínicas de la persona en diálisis que incluyó la adherencia a la toma de medicamentos, valorada con el Test de Morisky Green: instrumento validado para su uso en pacientes con enfermedades crónicas, el cual consta de cuatro preguntas que permiten conocer si el paciente cumple o no con la medicación ${ }^{14}$.

- Competencia para el cuidado versión-paciente con enfermedad crónica - versión abreviada. Conformada por 20 ítems medidos con una escala tipo Likert con opciones de respuesta casi nunca o nunca, pocas veces, con frecuencia, casi siempre o siempre, en la escala de 0 a 3 respectivamente. La calificación del instrumento se realiza de la siguiente manera: Conocimiento (bajo 0-3, medio 4-6, alto 7-9); Unicidad (bajo 0-6, medio 7-9, alto 10-12); Instrumental (bajo 0-3, medio 4-6, alto 7-9); Disfrutar (bajo 0-6, medio 7-9, alto 10-12); Antici- pación (bajo 0-2, medio 3-4, alto 5-6); Relación e Interacción (bajo 0-6, medio 7-9, alto 10-12) y, la calificación global (bajo 0-36, medio 37-48, alto 49-60). Este cuestionario ha sido validado para la población de pacientes con enfermedad crónica en Colombia con un alfa de Cronbach de 0,9115. Está compuesto por seis categorías bajo el acrónimo (CUIDAR): Conocimiento, relacionado con las nociones que se tienen sobre la enfermedad, el tratamiento y los cuidados diarios. Unicidad, las condiciones personales para afrontar y superar los problemas y desafíos a los que se enfrentan. Instrumental y procedimental, las habilidades y destrezas para llevar a cabo las tareas de cuidado, satisfaciendo las necesidades básicas. Disfrutar, grado de bienestar y satisfacción personal relacionada con la calidad de vida percibida. Anticipación, prevé las necesidades e identifica riesgos. Relación social e interacción, relaciones sociales y redes de apoyo disponibles que fortalecen el cuidado.

El análisis estadístico se realizó mediante la descripción de los resultados con frecuencias relativas y absolutas para las variables cualitativas y medidas de tendencia central y dispersión para las cuantitativas, según siguieran una distribución normal o no. Para identificar las variables asociadas al nivel de competencia, se comparó la distribución de las variables sociodemográficas y clínicas según el grado de competencia, mediante la prueba de $\mathrm{Chi}^{2}$ o el Test Exacto de Fisher. Se consideraron asociaciones significativas para $p<0,05$. Para la recolección de datos y el procesamiento de los mismos se utilizó una Hoja de cálculo en Excel y el paquete estadístico Stata 14.

El estudio fue aprobado por el Comité de Ética de la Unidad Renal Fundación Nefrouros Neiva, acta número 09, los participantes fueron contactados en la institución en el momento de asistencia a la consulta 0 al tratamiento, se les dio a conocer el objetivo de la investigación y previa firma del consentimiento informado las investigadoras realizaron la recolección de la información.

\section{Resultados}

Dentro de las variables sociodemográficas de los participantes $(n=200)$, la mayoría eran mujeres, con un promedio de edad 59 $\pm 13,89$ años, contaban con pareja, tenían escolaridad primaria, su principal dedicación era al hogar, tenían apoyo de cuidador del grupo familiar y 
Tabla 1. Competencia para el autocuidado en relación con las características sociodemográficas de las personas en diálisis ( $n=200$ ).

\begin{tabular}{|c|c|c|c|c|c|c|c|c|c|}
\hline \multicolumn{8}{|c|}{ Competencia para el Cuidado } & & \\
\hline \multirow[t]{2}{*}{ Variables } & \multicolumn{2}{|c|}{ Bajo } & \multicolumn{2}{|c|}{ Medio } & \multicolumn{2}{|c|}{ Alto } & \multirow{2}{*}{$\mathbf{p}$} & \multicolumn{2}{|c|}{ Total Poblacion } \\
\hline & $\mathbf{n}$ & $\%$ & $\mathbf{n}$ & $\%$ & $\mathbf{n}$ & $\%$ & & $\mathbf{n}$ & $\%$ \\
\hline \multicolumn{10}{|l|}{ Edad } \\
\hline $18-28$ años & 1 & 5 & 1 & 1 & 5 & 5 & \multirow{3}{*}{$0,073^{*}$} & 7 & 3,5 \\
\hline 29 - 59 años & 9 & 43 & 36 & 43 & 44 & 46 & & 89 & 44,5 \\
\hline 60 años o más & 11 & 52 & 47 & 56 & 46 & 49 & & 104 & 52 \\
\hline \multicolumn{10}{|l|}{ Género } \\
\hline Hombre & 8 & 38 & 44 & 52 & 47 & 49 & \multirow{2}{*}{$0,52^{*}$} & 99 & 49,5 \\
\hline Mujer & 13 & 62 & 40 & 48 & 48 & 51 & & 101 & 50,5 \\
\hline \multicolumn{10}{|l|}{ Estado Civil } \\
\hline Casado & 7 & 33 & 35 & 42 & 37 & 39 & \multirow{5}{*}{$0,68^{*}$} & 79 & 39,5 \\
\hline Separado & 1 & 5 & 10 & 12 & 4 & 4 & & 15 & 7,5 \\
\hline Soltero & 5 & 24 & 13 & 15 & 18 & 19 & & 36 & 18 \\
\hline Unión Libre & 5 & 24 & 14 & 17 & 22 & 23 & & 41 & 20,5 \\
\hline Viudo & 3 & 14 & 12 & 14 & 14 & 15 & & 29 & 14,5 \\
\hline \multicolumn{10}{|l|}{ Ocupación } \\
\hline No labora & 1 & 5 & 6 & 7 & 2 & 2 & \multirow{5}{*}{$0,38^{*}$} & 9 & 4,5 \\
\hline Labora & 5 & 24 & 26 & 31 & 30 & 32 & & 61 & 30,5 \\
\hline Estudiante & 0 & 0 & 0 & 0 & 1 & 1 & & 1 & 0,5 \\
\hline Hogar & 15 & 71 & 51 & 61 & 56 & 59 & & 122 & 61 \\
\hline Pensionado & 0 & 0 & 1 & 1 & 6 & 6 & & 7 & 3,5 \\
\hline \multicolumn{10}{|l|}{ Cuidador } \\
\hline No & 1 & 4,8 & 2 & 2,4 & 3 & 3,2 & \multirow{2}{*}{$0,705^{*}$} & 6 & 3 \\
\hline $\mathrm{Si}$ & 20 & 95,2 & 82 & 97,6 & 92 & 96,8 & & 194 & 97 \\
\hline \multicolumn{10}{|c|}{ Nivel Socioeconomico } \\
\hline Bajo & 15 & 71 & 60 & 71 & 50 & 53 & \multirow{3}{*}{$0,11^{*}$} & 125 & 62,5 \\
\hline Medio & 6 & 29 & 19 & 23 & 37 & 39 & & 62 & 31 \\
\hline Alto & 0 & 0 & 5 & 6 & 8 & 8 & & 13 & 6,5 \\
\hline
\end{tabular}

* Test Exacto de Fisher.

presentaban un nivel socioeconómico bajo. Con respecto a la competencia para el cuidado en relación con las características sociodemográficas no se identificaron asociaciones estadísticamente significativas (tabla $\mathbf{1}$ ).

En la tabla 2 se representa la competencia para el autocuidado en relación con las características clínicas de las personas en diálisis, donde se observó correlación estadísticamente significativa con la variable asistencia puntual a la terapia $(p=0,024)$ y cumplimiento en adherencia farmacológica $(p=0,001)$. Más del $50 \%$ de los participantes evidenciaron: ausencia de edemas, normotensión y permanencia en peso seco.

Con relación a la competencia global para el autocuidado en el hogar la mayoría de la población se ubica en nivel alto con un $47 \%$, y medio el $42 \%$; en cuanto a las seis dimensiones del cuidado cinco arrojaron en porcentaje superior nivel alto de competencia: conocimiento $72,5 \%$, anticipación $67,5 \%$, instrumental $51,5 \%$, unicidad $50,5 \%$, disfrutar $49 \%$ y en nivel medio una dimensión, relación e interacción con el 
Tabla 2. Competencia para el autocuidado en relación con las características clínicas de las personas en diálisis ( $n=200)$.

\begin{tabular}{|c|c|c|c|c|c|c|c|c|c|}
\hline \multicolumn{8}{|c|}{ Competencia para el Cuidado } & & \\
\hline \multirow[t]{2}{*}{ Variables } & \multicolumn{2}{|c|}{ Bajo } & \multicolumn{2}{|c|}{ Medio } & \multicolumn{2}{|c|}{ Alto } & \multirow{2}{*}{$\mathbf{p}$} & \multicolumn{2}{|c|}{ Total Población } \\
\hline & $\mathbf{n}$ & $\%$ & $\mathbf{n}$ & $\%$ & $\mathbf{n}$ & $\%$ & & n & $\%$ \\
\hline \multicolumn{10}{|l|}{ TRR } \\
\hline Hemodiálisis & 19 & 90 & 81 & 96 & 90 & 95 & \multirow{2}{*}{$0,53 *$} & 190 & 95 \\
\hline Diálisis Peritoneal & 2 & 10 & 3 & 4 & 5 & 5 & & 10 & 5 \\
\hline \multicolumn{10}{|l|}{ IMC } \\
\hline Bajo Peso & 3 & 14 & 8 & 9 & 6 & 6 & \multirow{4}{*}{$0,67^{*}$} & 17 & 8,5 \\
\hline Normal & 10 & 48 & 46 & 55 & 45 & 47 & & 101 & 50,5 \\
\hline Sobrepeso & 7 & 33 & 22 & 26 & 35 & 37 & & 64 & 32 \\
\hline Obesidad & 1 & 5 & 8 & 10 & 9 & 10 & & 18 & 9 \\
\hline \multicolumn{10}{|c|}{ Presencia de Edemas } \\
\hline Si & 11 & 52 & 23 & 27 & 29 & 31 & \multirow{2}{*}{$0,084^{*}$} & 63 & 31,5 \\
\hline No & 10 & 48 & 61 & 73 & 66 & 69 & & 137 & 68,5 \\
\hline \multicolumn{10}{|c|}{ Metas en Tensión Arterial } \\
\hline Normal & 11 & 52 & 50 & 60 & 49 & 52 & \multirow{2}{*}{$0,55^{*}$} & 110 & 55 \\
\hline Alterado & 10 & 48 & 34 & 40 & 46 & 48 & & 90 & 45 \\
\hline \multicolumn{10}{|c|}{$\begin{array}{l}\text { Asiste Puntualmente } \\
\text { a la terapia }\end{array}$} \\
\hline Si & 15 & 71 & 73 & 87 & 88 & 93 & \multirow{2}{*}{$0,024 *$} & 176 & 88 \\
\hline No & 6 & 29 & 11 & 13 & 7 & 7 & & 24 & 12 \\
\hline \multicolumn{10}{|l|}{$\begin{array}{l}\text { Mantenimiento } \\
\text { de Peso Seco }\end{array}$} \\
\hline Si & 12 & 57 & 67 & 80 & 72 & 76 & \multirow{2}{*}{$0,098^{*}$} & 151 & 75,5 \\
\hline No & 9 & 43 & 17 & 20 & 23 & 24 & & 49 & 24,5 \\
\hline \multicolumn{10}{|c|}{ Test de Morisky Green } \\
\hline Cumple & 10 & 48 & 67 & 80 & 82 & 86 & \multirow{2}{*}{$0,001^{*}$} & 159 & 79,5 \\
\hline No Cumple & 11 & 52 & 17 & 20 & 13 & 14 & & 41 & 20,5 \\
\hline
\end{tabular}

* Test Exacto de Fisher.

$58 \%$, el resto de las dimensiones en nivel medio de competencia registraron valores por encima del $25 \%$ (tabla 3).

\section{Discusión}

La caracterización sociodemográfica de la población estudiada coincide con lo descrito en la literatura: edad predominante mayor de 60 años $^{16}$, acorde con la creciente prevalencia de ERC dada por el deterioro fisiológico de la función renal, desde la tercera década de la vida, relacionada al incremento de factores de riesgo cardiovascular ${ }^{17}$ y a estilos de vida inadecuados ${ }^{18,19}$, razón importante para intervenir en la prevención de la insuficiencia renal a partir de edades tempranas ${ }^{16}$.

Algo más de la mitad de la población de estudio fueron mujeres, similar a lo descrito por González et al. ${ }^{17}$, quienes contaban con pareja: casados o en unión libre, aspecto muy importante y determinante en la superación de los obstáculos, desafíos y efectos de los tratamientos impuestos por la condición de salud, así lo plantea Costa et al. ${ }^{19}$; los cuales pueden influir y generar alteraciones físicas, sociales, psíquicas, emo- 
Tabla 3. Nivel de competencia global en relación con las dimensiones para el autocuidado CUIDAR $(n=200)$.

\begin{tabular}{lccccccc}
\hline \multirow{2}{*}{ Nivel de Competencia global } & \multicolumn{2}{c}{ Bajo } & \multicolumn{2}{c}{ Medio } & \multicolumn{3}{c}{ Alto } \\
& $\mathbf{n}$ & $\%$ & $\mathrm{n}$ & $\%$ & $\mathbf{n}$ & $\%$ \\
Dimensiones del cuidado & 21 & $\mathbf{1 1}$ & $\mathbf{8 4}$ & $\mathbf{4 2}$ & $\mathbf{9 5}$ & $\mathbf{4 7}$ \\
\hline Conocimiento & 0 & 0 & 55 & 27,5 & 145 & 72,5 \\
\hline Unicidad & 38 & 19 & 61 & 30,5 & 101 & 50,5 \\
\hline Instrumental & 12 & 6 & 85 & 42,5 & 103 & 51,5 \\
\hline Disfrutar & 29 & 14,5 & 73 & 36,5 & 98 & 49 \\
\hline Anticipación & 1 & 0,5 & 64 & 32 & 135 & 67,5 \\
\hline Relación e Interacción & 27 & 13,5 & 116 & 58 & 57 & 28,5 \\
\hline
\end{tabular}

Fuente: elaboración propia.

cionales y muchas veces pueden llevar al aislamiento y a cuadros depresivos que empeoran cuando la persona vive sola.

Prevalecieron las personas con bajo nivel educativo, circunstancia favorecedora del control inadecuado de patologías precursoras de ERC ${ }^{20}$, quienes al no comprender el proceso de la enfermedad es posible que presenten dificultad con el manejo de la dieta a seguir y el tratamiento farmacológico; al contrario de las personas con mayor conocimiento de su situación clínica en las que se espera mejor capacidad para cuidar de sí mismas $^{19}$.

La ocupación más frecuente de los participantes es el hogar, condición que incrementa la vulnerabilidad de las familias al empobrecerlas ${ }^{21}$, pues la ERC y las TRR son factores de incapacidad laboral; aspecto por el cual Julián-Mauro et al. ${ }^{22}$ recomendó a las personas activas, iniciar su tratamiento con terapias domiciliarias y luego continuar en los centros de diálisis a medida que se altere su autonomía o aumente la complejidad clínica.

En la población estudiada predominó el nivel socioeconómico bajo, que sumado al déficit de recursos se asocia a factores de riesgo para ERC, ubicados así en posición de fragilidad económica como lo reportan diversos autores $^{17,20,23}$. La mayoría contaban con cónyuge o hijo/hija como cuidador, siendo este un importante factor en el soporte emocional y del cuidado; las personas con apoyo familiar presentaron una mejor competencia para autocuidarse en el hogar, razón por la cual se recomiendan programas de atención para personas con ERC y sus cuidadores ${ }^{24}$, como estrategias de intervención.
En lo que respecta a las características clínicas se encontró una prevalencia de HTA y DM como antecedentes familiares, personales y etiología de ERC, resultado similar con varios estu$\operatorname{dios}^{17}$, siendo estas enfermedades los principales factores de riesgo para el desarrollo y progresión de la ERC ${ }^{25}$, reafirmando la presencia de múltiples factores de riesgo cardiovascular ${ }^{26}$ con elevada morbimortalidad para esta población; condiciones para las cuales plantea Choi Es y Lee, intervenir con educación individualizada o en pequeños grupos, donde se dé más interacción del paciente y el profesional sanitario ${ }^{27}$.

La mayoría de las personas con tiempo de permanencia en TRR superior a 2 años, presentaron competencia alta, media, y una menor proporción baja para el autocuidado; algo lógico según lo expresa Costa et al. ${ }^{19}$ con el tiempo y trayectoria de vida, la persona en condición de cronicidad se adapta al manejo de la enfermedad y ciertos padecimientos, reflejando que ha aprendido a vivir con ella. El grupo de baja competencia para su autocuidado podría estar en proceso de resiliencia donde la aceptación de sí mismo y de la vida aminora la percepción negativa y la preocupación por la enfermedad ${ }^{28}$; y por tanto, otro valioso aspecto a fortalecer para mejorar la competencia.

Se destacan en el estudio diversos factores de riesgo cardiovascular que afectan el proceso de salud enfermedad, como las cifras altas de tensión arterial, sobrepeso y obesidad, representados con el IMC y perímetro abdominal ${ }^{29}$, relacionados además con la génesis o deterioro de la función renal; aspectos importantes y susceptibles de intervención en forma estricta, para llevar las cifras de presión arterial o los diversos parámetros bioquímicos al normal exigido, y así evitar el deterioro de la función renal, como lo propone Martínez et al..$^{30}$

Igualmente se identificaron características relevantes acerca del cuidado y la adherencia de las personas, como son: asistencia puntual a terapia dialítica y cumplimiento del régimen terapéutico, valorado a través del test de Morisky Green ${ }^{31}$; presencia de edemas, mantenimiento del peso seco, y presencia de complicaciones asociadas a la TRR, que están asociados con la compe- 
tencia para el cuidado, aspectos que deben ser manejados por el equipo de salud para facilitar la comprensión y cumplimiento del régimen terapéutico, como lo propone Carrillo et al. ${ }^{15}$

En cuanto a la competencia para el autocuidado se identifica que la mayor parte de los participantes se encuentran en rangos alto y medio respectivamente, hallazgo similar a los reportados por Carrillo-González, Gloria Mabel, et al. ${ }^{32}$ y Aldana, Erika Alexandra, et al. ${ }^{33}$ donde esta competencia se encontró mayoritariamente entre los niveles de medio y alto; los cuales no llegan a ser óptimos, y demuestran competencia inapropiada para resolver las necesidades de atención de las personas en situación de cronicidad.

Respecto a las dimensiones, se identifica que el nivel más bajo reportado fue unicidad (condiciones personales) la cual evalúa características tanto internas como de personalidad, base fundamental para el afrontamiento de la enfermedad crónica y el rol de la persona enferma o cuidador. Como lo plantea Carrillo et al. ${ }^{14}$, esta valoración permite entender a la población, comprender los aspectos básicos a evaluar, y constituye una herramienta para orientar de forma precisa al equipo de salud hacia dónde dirigir el cuidado a la persona en TRR.

La baja dependencia y función mental intacta favorecen la autonomía de la persona en diálisis y son aspectos que pueden promover el alto nivel de competencia en la dimensión de conocimiento, como lo describe CarriIlo-González et al. ${ }^{32}$, en esta dimensión no hubo reporte de personas con baja competencia. El contar con el apoyo de un cuidador, representa un importante aspecto para hacer frente a problemas, retos y desafíos de la salud, que fortalecen la convivencia social y relaciones interpersonales ${ }^{19}$.

Se evidenció relación positiva estadísticamente significativa de la competencia para el autocuidado con las variables asistencia regular a las terapias programadas y cumplimiento con la adherencia farmacológica, condición prioritaria de intervenir oportunamente como lo propone Carrillo Gonzalez et al. ${ }^{15}$

Se identifican unicidad, disfrutar y relación social e interacción, con nivel bajo de competencia y respuestas no favorables en los diferentes items, similar a lo encontrado en el estudio de la IPS Dialy-ser ${ }^{33}$, aspectos relacionados a formas de afrontamiento de los usuarios frente a su enfermedad que pueden afectar la calidad de vida de las personas ${ }^{20}$.
En algunos estudios, el afrontamiento dirigido al problema constituye una estrategia favorable a la adhesión al tratamiento en personas hemodializadas, y promueve la adaptación positiva del paciente al tratamiento ${ }^{34}$. Según el estudio de Benett P. ${ }^{35}$ el bienestar subjetivo puede ser un indicador importante de la calidad de vida que debe ser estudiado y tratado.

La dimensión de relación social e interacción, reportó nivel de competencia baja de autocuidado, escenario de gran importancia de gestionar para Costa et al. ${ }^{19}$, teniendo en cuenta que la enfermedad lleva al aislamiento del paciente y a cuadros depresivos.

La familia y los amigos son las redes de apoyo base como estrategias de adaptación y afrontamiento a la diálisis y enfermedad, son vitales para el día a día de estos individuos.

Enfocados desde el cuidado y atención de Enfermería de la persona y sus necesidades, lo anterior representa evidencia para plantear intervenciones en la práctica e investigación y aportar al mejoramiento en la cualificación del cuidado enfermero.

Esta investigación tiene como limitación el empleo de un muestreo por conveniencia, situación que no hace posible la generalización de los resultados de la investigación. De igual manera el número de participantes en tratamiento de diálisis peritoneal, es bajo en comparación con las personas en tratamiento de hemodiálisis.

A partir de los resultados observados podemos concluir que el perfil de la persona con ERC en tratamiento con diálisis es semejante a lo expuesto por la literatura en relación a las similitudes con otras condiciones crónicas de salud. Se evidencia una prevalencia en aumento en el género femenino del grupo estudiado, así como en usuarios con bajos niveles de escolaridad, ocupación hogar y de nivel socioeconómico bajo. La competencia del cuidado se caracterizó por estar entre los niveles de alta y media, se observó relación estadísticamente significativa con las características clínicas, asistencia puntual a terapia y cumplimiento en adherencia farmacológica.

Aunque, la mayoría de la población presentó un nivel de competencia para el autocuidado alto, es necesaria la intervención fundamental del profesional de Enfermería y del equipo de salud con estrategias participativas que impacten en el empoderamiento que los lleven a asumir cambios en el comportamiento y conductas permanentes para fomentar el mejor nivel de competencia 
de cuidado, adherencia a los mismos, evitar complicaciones y lograr mejores desenlaces en salud; con la meta de eliminar el déficit entre estas capacidades de autocuidado y la demanda terapéutica.

Recepción: 06-06-20

Aceptación: 15-09-21

Publicación: 30-12-21

\section{Bibliografía}

1. Ayar Y, Ersoy A, Ocakoglu G, Yildiz A, Oruc A, Soyak $H$, et al. Risk Factors Affecting Graft and Patient Survivals After Transplantation From Deceased Donors in a Developing Country: A Single-Center Experience. Transplant Proc [Internet]. 2017 Mar [consultado 15 Sep 2020];49(2):270-7. Disponible en: https://www.sciencedirect.com/science/article/abs/pii/ S004113451630954X.

2. Schaepe C, Bergjan M. Educational interventions in peritoneal dialysis: a narrative review of the literature. Int J Nurs Stud. 2015 Apr;52(4):882-98. D0I: https:// doi,org/10.1016/j.ijnurstu.2014.12.009. Epub 2015 Jan 5. PMID: 25616708.

3. Cruz VFES da, Tagliamento G, Wanderbroocke AC. A manutenção da vida laboral por doentes renais crônicos em tratamento de hemodiálise: uma análise dos significados do trabalho. Saúde e Soc [Internet]. 2016 Dec [consultado 15 Sep 2020];25(4):1050-63. Disponible en: http://www.scielo.br/scielo.php?script=sci_arttext\&pid=S0104-12902016000401050\&Ing=pt\&tIn$g=p t$.

4. ERC más de 850 millones de personas en el mundo la padecen - CONSULTORSALUD [Internet]. [consultado 4 Abr 2021]. Disponible en: https://consultorsalud. com/erc-mas-de-850-millones-de-personas-en-el-mundo-la-padecen/.

5. Bergjan M, Schaepe C. Educational strategies and challenges in peritoneal dialysis: A qualitative study of renal nurses experiences. $\mathrm{J}$ Clin Nurs [Internet].
2016;25(11-12):1729-39. Disponible en: http://search. proquest.com/docview/1793579178?accountid=17192.

6. Tejada Tayabas LM, Partida Ponce KL, Hernández-Ibarra LE. Coordinated hospital-home care for kidney patients on hemodialysis from the perspective of nursing personnel. Rev Lat Am Enfermagem [Internet]. 2015 [consultado 23 may 2018];23(2):5-33.Disponible en: http:// www.scielo.br/scielo.php?script=sci_arttext\&pi$d=$ S0104-11692015000200007\&lng=en\&tlng=en .

7. Cho MK, Shin G. Gender-based experiences on the survival of chronic renal failure patients under hemodialysis for more than 20 years. Appl Nurs Res [Internet]. 2016 [cited 2017 Apr 12];32:262-8.

8. Velázquez Pérez Y, Espín Andrade AM. Repercusión psicosocial y carga en el cuidador informal de personas con insuficiencia renal crónica terminal. Rev Cubana de Salud Pública. 2014;1(40):3-17. [consultado 17 Nov 2021]. Disponible en: http:// scielo.sld.cu/scielo.php?script=sci_arttext\&pi$\mathrm{d}=$ S0864-34662014000100002.

9. Tejada Tayabas LM, Partida Ponce KL, Hernández Ibarra LE. Coordinated hospital-home care for kidney patients on hemodialysis from the perspective of nursing personnel. Rev Lat Am Enfermagem [Internet]. 2015 Apr [consultado 15 Sep 2021];23(2):225-33. Disponible en: http://www.scielo.br/scielo.php?scrip$\mathrm{t}=$ sci_arttext\&pid=S0104-11692015000200007\&ln$g=e n \&$ tIng=en.

10. Aldana MSC, Durán-Parra M, Solano-Aguilar S, Moya-Plata D, Salazar LBA, Duran Niño EY, et al. Costos derivados de la atención a pacientes con enfermedad crónica no transmisible. Respuestas [Internet]. 2014 Jul [consultado 1 Abr 2021];19(2):51-8. Disponible en: https://revistas.ufps.edu.co/index.php/respuestas/ article/view/497/514.

11. Barrera Ortiz L, Vargas Rosero E, Cendales PA. Encuesta de caracterización para el cuidado de una persona con enfermedad crónica. Investig en Enfermería Imagen y Desarro [Internet]. 2014 Dec 15 [consultado 23 May 2021];17(1):27-43. Disponible en: https://www.researchgate.net/publication/285123325_Encuesta_de_caracterizacion_para_el_cuidado_de_una_persona_con_enfermedad_cronica/link/5708095a08ae8883alf7f1f8/ download. 
12. Bermejo Pareja F, Porta Etessam J, Díaz Guzmán J, Martínez Martín P. Mas de cien escalas en neurología [Internet]. Aula Medica, 2da ed. Toledo; 2008 [consultado 2 Sep 2021]. p. 376. Disponible en: http://www. neuroloxia.com/wp-content/uploads/2009/06/escalas_ en_neurologia_marzo.pdf

13. Martínez de la Iglesia J, Dueñas Herrero R, Onís Vilches M, Aguado Taberné C, Albert Colomer C, Luque Luque R. Adaptación y validación al castellano del cuestionario de Pfeiffer (SPMSQ) para detectar la existencia de deterioro cognitivo en personas mayores de 65 años. Med Clin (Barc) [Internet]. 2001 [consultado 2 Sep 2021];117(4):129-34. Disponible en: https://medes. com/publication/3015.

14. Morisky DE, Green LW, Levine DM. Concurrent and Predictive Validity of a Self-reported Measure of Medication Adherence. Med Care [Internet]. 1986 Jan [consultado 2 Sep 2021];24(1):670-74. Disponible en: http://www.ncbi.nlm.nih.gov/pubmed/3945130.

15. Carrillo González GM, Sánchez Herrara B, Vargas Rosero E. Desarrollo y pruebas psicométricas del Instrumento "cuidar" - versión corta para medir la competencia de cuidado en el hogar. Rev la Univ Ind Santander Salud. 2016;48(2):222-31.

16. Bunch Barrera A, Tamer David LM, Ardila Celis F, Laganis Valcarcel S, Castaño Rodríguez R, Vesga Gualdrón JI, et al. Impacto de un modelo de gestión de enfermedad en una población con tratamiento de diálisis en Colombia. Rev la Fac Med. 2016;64(4):695.

17. González BS, Pascual MR, Guijarro LR, González AF, Puertolas OC, Latre LMR. Enfermedad renal crónica en Atención Primaria: Prevalencia y factores de riesgo asociados. Aten Primaria. 2015 Apr;47(4):23645.

18. Chowdhury R, Peel NM, Krosch M, Hubbard RE. Frailty and chronic kidney disease: A systematic review. Arch Gerontol Geriatr [Internet]. 2017 [consultado 7 sep 2021]; 135-42. Disponible en: https://reader. elsevier.com/reader/sd/pii/S016749431630187X?token=D4A3F24DF1C236F88ED9D0B768110C7 2C9F2D4F14B30F81AB115AD502CCOE51A90ED22A96E063D062235338A683C567E\&originRegion=eu-west-1\&originCreation=20211221083823.

19. Costa GMA, Pinheiro MBGN, Medeiros SM de, Costa RR de 0, Cossi MS. Calidad de vida en pacientes con in- suficiencia renal crónica en hemodiálisis. Enferm Glob. 2016 Jun;15(3):59-99.

20. Pérez Acuña C, Riquelme Hernández G, Scharager Goldenberg J, Armijo Rodríguez I. Relación entre calidad de vida y representación de enfermedad en personas con enfermedad renal crónica terminal en tratamiento con hemodiálisis. Enferm Nefrol. 2015;2:89-96.

21. Bona K, Dussel V, Orellana L, Kang T, Geyer R, Feudtner $\mathrm{C}$, et al. Economic Impact of Advanced Pediatric Cancer on Families. J Pain Symptom Manage [Internet]. 2014 Mar [consultado 21 Ago 2021];47(3):594-603. Disponible en: https://www.sciencedirect.com/science/ article/pii/S0885392413003102.

22. Julián Mauro JC, Molinuevo Tobalina JÁ, Sánchez González JC. La situación laboral del paciente con enfermedad renal crónica en función del tratamiento sustitutivo renal. Nefrologia [Internet] 2012 [consultado 16 Jun 2021];32(4):439-45. Disponible en: http://scielo.isciii. es/scielo.php?pid=S0211-69952012000600004\&script=sci_arttext\&tlng=en.

23. García García G. La pobreza: el denominador común de la amenaza mundial de la ERC [Internet]. Vol. 16, MEDICC Review. 2014 [consultado 17 Jun 2021]. Disponible en: https://www.medigraphic.com/pdfs/medicreview/mrw-2014/mrw142q.pdf.

24. Arias Rojas M, Barrera Ortiz L, Carrillo GM, Chaparro Díaz L, Sánchez Herrera B, Vargas Rosero E. Cuidadores familiares de personas con enfermedad crónica en las regiones de frontera colombiana: perfil y carga percibida de cuidado. Rev la Fac Med. 2015 Feb 10;62(3):387-97.

25. Navarro González J, Mora Fernández C, Martínez Castelao A, Gorriz Teruel JL, Soler Romeo MJ, de Alvaro Moreno F. Enfermedad renal diabética: etiopatogenia y fisiopatología. En: Nefrología al día. Sociedad Española de Nefrología 2020 [consultado 3 Nov 2021]. Disponible en: https://www.nefrologiaaldia.org/264.

26. Subiza AK, Odriozola M, Ríos $P$, Ana D, Subiza K, Lamadrid $V$, et al. Riesgo cardiovascular en la enfermedad renal crónica. Rev Uruguaya Cardiol. 2016;31(2):20618.

27. Choi, Eun Sungl Lee J. Effects of a Face-to-face Self-management Program on Knowledge, Self-care Practice and Kidney Function in Patients with Chronic 
Kidney Disease before the Renal Replacement Therapy. $2012 ; 42(7): 1070-8$.

28. González Flores C, Ureña Rodríguez M, Meda Lara R. Resiliencia y enfermedad renal crónica: Una Revisión sistemática. RIP [Internet]. 2018 Dic [consultado 17 Sep 2021];11(2):79-6. Disponible en: https://reviberopsicologia.ibero.edu.co/article/view/ rip.11209/pdf.

29. Otero González A, de Francisco ALM, Gayoso P, López FG. Obesidad y función renal. Datos del estudio epidemiológico: Prevalencia de la enfermedad renal crónica en España. Estudio EPIRCE. Nefrología. 2018 Jan;38(1):107-8.

30. Martínez Castelao A, Górriz Teruel JL, Bover Sanjuán J, Segura-de la Morena J, Cebollada J, Escalada J, et al. Consensus document for the detection and management of chronic kidney disease. Nefrologia [Internet]. 2014 [consultado 20 Abr 2020];34:24362. Disponible en: https://revistanefrologia.com/ es-documento-consenso-deteccion-manejo-enfermedad-articulo-X0211699514053919.

31. Pisano González M M, González Pisano AC. La modificación de los hábitos y la adherencia terapéutica, clave para el control de la enfermedad crónica. Jan 1, 2014. p. 59-66.
32. Carrillo González GM, Barreto RV, Arboleda LB, Gutierrez-Lesmes OA, Gregoria-Melo B, Tamara-Ortiz V. Competencia para cuidar en el hogar de personas con enfermedad crónica y sus cuidadores en Colombia. Rev la Fac Med. 2015; 63(4):669-75.

33. Aldana EA, Barrera SY, Rodríguez KA, Gómez 0J, Carrillo GM, Yomaira S, et al. Competencia para el cuidado (CUIDAR) en el hogar de personas con enfermedad renal crónica en hemodiálisis. Enfermería Nefrológica. 2016; 19(3):3-9.

34. Khechane NL, Mwaba K. Treatment adherence and coping with stress among black south african haemodialysis patients. Soc Behav Pers. 2004 Jan 1; 32(8):777-82.

35. Bennett PN, Weinberg MK, Bridgman T, Cummins RA. The happiness and subjective well-being of people on haemodialysis. J Ren Care. 2015 Sep 1; 41(3):156-61.

Este artículo se distribuye bajo una Licencia Creative Commons Atribución-NoComercial 4.0 Internacional. https://creativecommons.org/licenses/by-nc/4.0/ 